\title{
El acaparamiento del agua: un problema global
}

La expansión de sistemas de producción que requieren un uso muy intensivo de materiales, energía y agua, $y$ que generan residuos y contaminación.

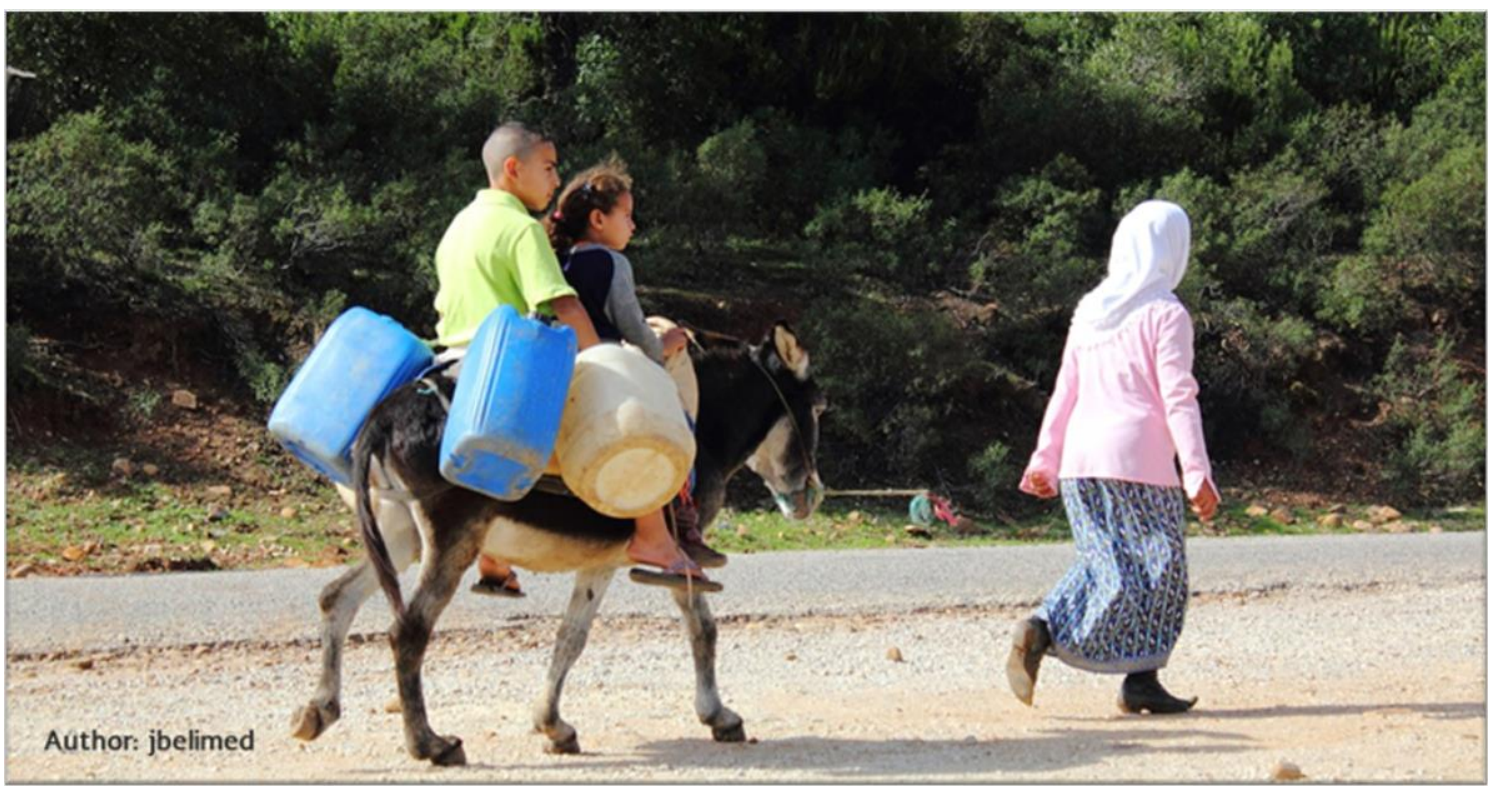

El siglo XXI inició en una situación definida por sociedades que deben adaptarse a un mundo en constante cambio.

Con recursos naturales limitados, con un acelerado incremento poblacional y con la expansión de sistemas de producción que requieren un uso muy intensivo de materiales, energía y agua, y que generan residuos y contaminación (Rockström et al., 2009; Ravera et al., 2014). En este contexto, que incrementa la necesidad de alimentos, combustibles y fibras, las grandes corporaciones agroindustriales han aumentado, a su vez, la presión que ejercen sobre la Tierra y sus recursos, particularmente en países en vías de desarrollo, donde las amplias áreas agrícolas se consideran de bajo desempeño.

Existe un movimiento documentado de empresas transnacionales que se han concentrado en adquirir tierra en países en vías de desarrollo (p. ej., Cotula, 2009, Byerlee y Deininger, 2013). Este fenómeno, bautizado como "acaparamiento de tierra" o "fiebre global por la tierra", ha llamado la atención de los medios de comunicación, organizaciones de la sociedad civil e instituciones académicas. Esto ha dado lugar a un cuerpo emergente de conocimiento que estudia y analiza las transformaciones asociadas a las adquisiciones de tierra de gran escala (ATCE) y a la dinámica de poder que resulta del acceso/uso de 
la tierra y sus recursos (p. ej., Borras et al., 2011, De Schutter, 2011, Cotula, 2013). Los trabajos más recientes han presentado resultados relativos a los impactos de este fenómeno sobre el bienestar de las comunidades rurales (Davis et al., 2014, D'Odorico y Rulli, 2014, Oberlack et al., 2016), la seguridad alimentaria (D'Odorico y Rulli, 2013) y el desarrollo sustentable (Dell'Angelo et al., 2018). Adicionalmente, se han identificado como factores determinantes en el impulso de este fenómeno a la especulación financiera (Fairhead et al., 2012) y a la producción de energía (Scheidel y Sorman, 2012).

Sin embargo, como resultado del trabajo inquisitivo de investigación sobre el ATGE, ha surgido de forma natural una hipótesis alternativa: ¿Es posible que esta fiebre mundial por la adquisición de tierra sea resultado de la necesidad de agua, más que de la necesidad de tierra en sí misma? (Skinner y Cotula, 2011, Allan et al., 2012, GRAIN, 2012, Mehta et al., 2012, Woodhouse, 2012, Franco et al., 2013).

El agua es un elemento natural que, como sabemos, es clave para el desarrollo económico de todas las sociedades. En el mundo globalizado, un mecanismo poco estudiado de apropiación de agua es el que está asociado justamente a las grandes inversiones de la agroindustria. Entender el problema de la adquisición de tierra a través de un lente hidrológico nos provee una manera alternativa de evaluar los impactos que resultan de la compra de grandes extensiones de tierra y de los países que hacen y reciben la inversión económica. Para ello, se usan conceptos como el de huella hídrica, abordado en este blog, que nos permite entender la dinámica de la globalización y su impacto sobre el agua (Rulli et al., 2013, Rulli and D'Odorico, 2013, Breu et al., 2016).

Rulli et al. (2013) presentaron una primera evaluación de la apropiación global del agua a través de la ATGE, estimando la cantidad de agua apropiada a través de la producción de cultivos en tierras adquiridas, así como sus efectos potenciales en la seguridad alimentaria de los países que reciben la inversión económica. En otras instancias, el término de acaparamiento de agua se utiliza para identificar la apropiación directa de agua en los territorios, por ejemplo, por actividades relativas a la generación de energía eléctrica (Islar, 2012) o a la minería (Sosa y Zwarteveen, 2012). De hecho, existe cierta ambigüedad en la manera en la que se define al acaparamiento del agua en la literatura. En parte, esta confusión se debe a que el término se ha utilizado para referirse a diferentes formas de apropiación de agua y para describir distintas dinámicas.

\section{¿Qué es el acaparamiento de agua?}

Como se ha dicho, el concepto de acaparamiento de agua se ha utilizado para describir diferentes fenómenos. El común denominador entre las definiciones incluye aspectos relativos a la injusticia y a un desequilibrio de poder entre los diferentes actores dentro de un territorio. Así, el acaparamiento de agua determina algo diferente de la apropiación, explotación, extracción, consumo y uso de agua. Involucra la noción de un "acaparador" y un "despojado"; es decir, una dinámica de usurpación que tiene su base en un desequilibrio de poderes entre los sujetos que pierden y los que ganan de forma injusta. La definición de acaparamiento de agua se ocupa de la cuestión ética de cuándo es apropiado definir un caso particular de tipología de extracción de agua como "acaparamiento". También se enfoca en la pregunta biofísica de cómo cuantificamos o identificamos la apropiación de un elemento que por su naturaleza es fluido, renovable y difícil de cuantificar (Rodríguez-Labajos y Martínez-Alier, 2015).

El esfuerzo más completo de sistematización de las definiciones de este concepto está registrado en el número especial de Water Alternatives (Mehta et al., 2012), con 14 artículos que lo abordan desde diferentes perspectivas. El común denominador, una vez más, son las situaciones de desequilibrio de poder en la apropiación de agua, generalmente en perjuicio de los usuarios locales y sus derechos consuetudinarios. Mehta et al. (2012) definen al acaparamiento de agua como una situación en la que 
PERSPECTIVAS IMTA (0)

$\mathrm{N}^{\circ} .24,2020$

MEDIO AMBIENTE

Autor: Adrián Pedrozo Acuña

DOI: doi.org/10.24850/b-imta-perspectivas-2020-24

actores poderosos son capaces de tomar control del agua y reasignar para su propio beneficio los derechos utilizados por comunidades locales y ecosistemas acuáticos que sustentan su vida.

El concepto de huella hídrica (cantidad de agua utilizada para crear un bien o servicio) se define de forma típica para los usos consuntivos del agua. Es por ello que este concepto puede ser utilizado para referirse a las apropiaciones y acaparamiento de agua que implican un uso consuntivo (p. ej., agricultura, minería, etc.). Más aún, en algunos casos, los usos no consuntivos pueden ser también incluidos en virtud de que el agua es regresada al ambiente con una calidad deteriorada, a una elevación diferente o en otra ubicación.

\section{¿Qué tipo de agua es acaparada?}

El agua dulce del planeta se encuentra almacenada en los casquetes polares, bajo tierra (p. ej. en los acuíferos), en el suelo somero (p. ej. humedad del suelo de la zona de raíces de la mayoría de las plantas) y en los cuerpos de agua superficiales (p. ej., ríos, lagos, lagunas y embalses).

Los casquetes polares no contribuyen a la mayoría de los usos productivos del agua hasta que el hielo es derretido y se transforma en agua superficial y subsuperficial. La humedad del suelo en la zona de raíces, conocida también como agua verde (Falkenmark et al., 1989), es solamente aprovechada por las plantas y los organismos que habitan en el suelo, y contribuye, por tanto, a la productividad de los ecosistemas terrestres. El agua almacenada en los acuíferos y cuerpos de agua superficiales es definida como agua azul, y puede ser extraída en grandes cantidades por medio de pozos, canales y tuberías para ser conducida a fábricas, campos agrícolas, comunidades y ciudades. Así, mientras que el agua verde está atada a la tierra, el agua azul puede ser transportada. La agricultura puede depender exclusivamente del agua verde (p. ej. temporal) o de ambas: verde y azul (p. ej. riego). De esta forma, la adquisición de tierra para cultivo está directamente asociada con la apropiación de agua verde, aunque si los inversionistas construyen infraestructura de riego, también es probable que se apropien de agua azul. En otras palabras, el acaparamiento de tierra es también acaparamiento de agua verde, con potencial de convertirse en acaparamiento de agua azul, si los inversionistas riegan la tierra.

El vínculo entre la escasez hídrica y la seguridad alimentaria es evidente: la agricultura de riego representa el mayor consumidor de agua azul, con cerca del $69 \%$ de las extracciones anuales globales y el $92 \%$ del consumo (Richter, 2014).

Entender las implicaciones hidrológicas del vínculo entre agua y producción de alimentos, que además se da como resultado de las ATGE y la exportación/importación de bienes y productos, es una de las actividades más importantes para los investigadores del sector hídrico nacional. A pesar de la dificultad que pueda implicar evaluar la dimensión hídrica de problemas asociados con la tenencia de la tierra y su apropiación, esto deberá ser materia de estudio, dada la realidad que apreciamos en el mundo (Breu et al., 2016). Una vía necesaria, ya identificada por este gobierno, es andar hacia decisiones más transparentes, con base ética, incluyentes y participativas.

Nuestra responsabilidad, como Instituto Mexicano de Tecnología del Agua, es proveer las herramientas y métodos con base en conocimiento científico de punta, que permitan la construcción de un marco de trabajo que identifique la probabilidad de acaparamiento de agua (verde y azul), sea como resultado de la presencia de ATGE o por el uso de conocimiento anticuado. 
PERSPECTIVAS IMTA (0)

$N^{\circ} .24,2020$

La importancia de atender este tema estriba en que el despojo de agua de las comunidades rurales o periurbanas en favor de grandes usuarios agrícolas o industriales tiene el potencial de convertirse en un límite a la producción económica. Para evitarlo es necesario garantizar el acceso al agua de las comunidades y el uso sustentable por parte de los grandes usuarios que comparten el mismo territorio. Esta es una razón más para incluir la ética en las decisiones en torno al agua.

De hecho, el acaparamiento de agua azul puede ser definido como una apropiación no ética de agua, considerándola poco ética cuando ésta se realiza en regiones con escasez hídrica y erosiona los derechos humanos. La literatura global sobre ATGE intenta responder a la pregunta clave de si este tipo de sistemas de gran escala tiene un impacto positivo o negativo sobre la seguridad alimentaria de los países con altos índices de desnutrición y escasez de agua (Schiffman, 2013). La misma pregunta es relevante para el estudio del acaparamiento de agua. En general, es poco claro si la transformación agraria de pequeños productores con sistemas tradicionales hacia sistemas industrializados ha tenido un impacto positivo en la seguridad alimentaria de países o regiones con escasez hídrica.

El fenómeno emergente de acaparamiento de agua nos impone la necesidad de dedicar mayores esfuerzos de investigación a la generación de conocimiento de punta y su utilización en la toma de decisiones. Es vital que recorramos este camino con un paso más acelerado para hacer del agua el motor de desarrollo económico equitativo que México requiere.

\section{Referencias:}

Allan T., M. Kerlertz, S. Sojamo, J. Warner, Handbook of Land and Water Grabs in Africa: Foreign Direct Investment and Water and Food Security, Routledge, London (2012).

Borras Jr. S.M., et al. Towards a better understanding of global land grabbing: an editorial introduction, J. Peasant Stud., 38 (2) (2011), pp. 209-216.

Breu T., C. Bader, P. Messerli, A. Heinimann, S. Rist, S. Eckert, Large-scale land acquisition and its effects on the water balance in investor and host countries, PLoS One, 11 (3) (2016).

Byerlee, D., K. Deininger Growing resource scarcity and global farmland investment, Ann. Rev. Resour. Econ., 5 (1) (2013), pp. 13-34.

De Schutter O., Green Rush: The Global Race for Farmland and the Rights of Land Users, 52, The. Harv Int'I LJ (2011), p. 503.

Cotula, L. (2009) Land Grab or Development Opportunity? Agricultural Investment and International Land Deals in Africa.

Cotula, L. (2013), The new enclosures? Polanyi, international investment law and the global land rush, Third World Q., 34 (9) pp. 1605-1629.

Davis K.F., P. D'Odorico, M.C. Rulli Land grabbing: a preliminary quantification of economic impacts on rural livelihoods, Popul. Environ., 36 (2) (2014), pp. 180-192.

Dell'Angelo, J., M.C. Rulli, P. D'Odorico, Threats to sustainable development posed by land and water grabbing,

Curr. Opin. Environ. Sustain. (2018).

D'Odorico, P., M. Rulli, The land and its people, Nature Geoscience, 7 (5) (2014), pp. 324-325, 10.1038/ngeo2153

D'Odorico, P., M. Rulli, The fourth food revolution, Nat. Geosci., 6 (6) (2013), pp. 417-418.

Fairhead J., M. Leach, I. Scoones, Green grabbing: a new appropriation of nature? J. Peasant Stud., 39 (2) (2012), pp. 237-261.

Falkenmark M., J. Lundquist, C. Widstrand, Macro-scale water scarcity requires micro-scale approaches: Aspects of vulnerability in semi-arid development, Nat. Res. Forum, 13 (4) (1989), pp. 258-267.

Franco J., L. Mehta, G.J. Veldwisch, The global politics of water grabbing, Third World Q., 34 (9) (2013), pp. 1651-1675 GRAIN Squeezing Africa dry: Behind Every Land Grab is a Water Grab, Barcelona. Available online at

https://www.grain.org/article/entries/4516-squeezing-africa-dry-behind-every-land-grab-is-a-water-grab (2012). Islar M., Privatised hydropower development in Turkey: a case of water grabbing? Water Altern., 5 (2) (2012), p. 376 Mehta, L., G.J. Veldwisch, J. Franco Introduction to the Special Issue: water grabbing? Focus on the (re)appropriation of finite water resources, Water Altern., 5 (2) (2012), p. 193. 
PERSPECTIVAS IMTA (0)

$N^{\circ} .24,2020$

Autor: Adrián Pedrozo Acuña

\section{DOI: doi.org/10.24850/b-imta-perspectivas-2020-24}

Oberlack, C., L. Tejada, P. Messerli, S. Rist, M. Giger, Sustainable livelihoods in the global land rush? Archetypes of livelihood vulnerability and sustainability potentials, Glob. Environ. Chang., 41 (2016), pp. 153-171.

Ravera, F. , A. Scheidel, J. Dell'Angelo, G. Gamboa, T. Serrano, S. Mingorría, V. Cabello, N. Arizpe, P. Ariza, Pathways of rural change: an integrated assessment of metabolic patterns in emerging ruralities, Environ. Dev. Sustain., 16 (4) (2014), pp. 811-820.

Rockström, J., W. Steffen, K. Noone, A. Persson, F.S. Chapin, E.F. Lambin, T.M. Lenton, M. Scheffer, C. Folke, H.J. Schellnhuber, B. Nykvist. A safe operating space for humanity, Nature, 461 (7263) (2009), pp. $472-475$

Rodríguez-Labajos B., J. Martínez-Alier, Political ecology of water conflicts Wiley Interdisciplinary Reviews: Water, 2(5) (2015), pp. 537-558.

Richter B., Chasing Water, Island Press, Washington, DC (2014).

Rulli, M.C., A. Saviori, P. D'Odorico, Global land and water grabbing, Proc. Natl. Acad. Sci., 110 (3) (2013), pp. 892-897 Rulli, M.C., P. D'Odorico, The water footprint of land grabbing, Geophys. Res. Lett., 40 (23) (2013), pp. 6130-6135

Scheidel, A., A.H. Sorman, Energy transitions and the global land rush: ultimate drivers and persistent consequence, Glob. Environ. Chang., 22 (3) (2012), pp. 588-595.

Schiffman R., Hunger, food security, and the African land grab, Ethics Int. Aff., 27 (3) (2013), pp. 239-249

Skinner, J., L. Cotula, Are land deals driving 'water grabs' Briefing: The Global Land Rush, IIED, London

(2017) http://pubs.iied.org/1702IIED.html

Sosa M., M. Zwarteveen, Exploring the politics of water grabbing: the case of large mining operations in the Peruvian Andes, Water Altern., 5 (2) (2012), p. 360.

Woodhouse P., Foreign agricultural land acquisition and the visibility of water resource impacts in Sub-Saharan Africa Water Altern., 5 (2) (2012), p. 208. 